CASE REPORT

\author{
K. Yamada \\ K. Sakai \\ K. Owada \\ K. Mineura \\ T. Nishimura
}

\section{Cerebral White Matter Lesions May Be Partially Reversible in Patients with Carotid Artery Stenosis}

SUMMARY: Contrary to the common belief that age-related WMLs (also known as leukoaraiosis) are a progressive condition, a case of partial reversal of WMLs shortly after carotid artery stenting is described. A 75-year-old man presented with frequent TIAs, which were attributed to right ICA stenosis. He subsequently underwent successful carotid artery stenting. Follow-up MR imaging a week after the procedure showed improvement of WMLs in the right cerebral hemisphere. Pixel-bypixel image analysis showed that the reversed WMLs tended to have higher $\lambda 1$ and lower signal intensity on $b=0$ images compared with nonreversed lesions, but by only approximately $10 \%$.
C erebral WMLs can often be detected on CT and MR imaging in elderly individuals. The term "leukoaraiosis" is often used to describe these WMLs. ${ }^{1}$ Epidemiologic studies have shown that these WMLs are correlated with age, hypertension, arteriosclerosis, cigarette smoking, and glucose intolerance. ${ }^{2,3}$ These predisposing factors are also found in patients with lacunar infarcts; thus, it has been proposed that WMLs and lacunar infarcts share a similar underlying vasculopathy, namely, subcortical occlusive small-vessel disease secondary to arteriolosclerosis. These age-related WMLs are considered to be a progressive process. ${ }^{3,4}$ In fact, to our knowledge, improvement of such ischemic lesions in a short period of time has not been well documented. We report herein a case with partial resolution of WMLs shortly after restoration of blood flow by carotid artery stenting. We conducted a retrospective image analysis on the pre- and postprocedural MR images to determine if there were any differences between these WMLs, hoping that this would cast some light on their pathophysiology.

\section{Case Report}

A 75-year-old man presented to our hospital with TIAs, characterized by intermittent left upper extremity weakness. DWI revealed no apparent acute infarction. Motion-sensitizing gradients of this DWI were applied to 15 different directions to enable DTI. Punctate WMLs were noted on FLAIR images at the centrum semiovale of both cerebral hemispheres, with right hemispheric laterality (Fig 1A). MR angiography revealed severe right ICA stenosis with distal flow compromise (Fig $1 B)$.

Received July 22, 2009; accepted after revision August 5.

From the Departments of Radiology (K.Y., T.N.) and Neurosurgery (K.O., K.M.), Graduate School of Medical Science, Kyoto Prefectural University of Medicine, Kyoto City, Kyoto, Japan; and Graduate School of Medicine (K.S.), Faculty of Human Health Sciences, Kyoto University, Kyoto City, Kyoto, Japan.

Please address correspondence to Kei Yamada, MD, Department of Radiology, Kyoto Prefectural University of Medicine, Kajii-cho, Kawaramachi Hirokoji Agaru, Kamigyo-ku, Kyoto City, Kyoto 602-8566, Japan; e-mail: kyamada@koto.kpu-m.ac.jp

DOI 10.3174/ajnr.A1873
Subsequent right carotid conventional angiography revealed pseudo-occlusion of the right ICA, with marked delay in contrast arrival at the right cerebral hemisphere. A successful stent-placement procedure was performed without any notable complications. Follow-up MR imaging 7 days after the procedure showed a patent right ICA and improvement of WMLs in the right cerebral hemisphere (Fig $1 C,-D)$. The patient remained asymptomatic at the recent 18 -month follow-up.

To characterize the reversed WMLs, we performed a retrospective image analysis on the MR imaging data. First, affine registration of pre- and postprocedural FLAIR images was carried out, after which segmentation of the WMLs was performed (Fig $2 A,-B$ ). Subtraction images yielded pixels with/without reversal (Fig $2 C$ ). We coregistered these data onto the DWI/DTI to characterize the nature of the WMLs. A $t$ test was used for statistical analysis, and $P$ values $<.05$ were considered significant.

This image analysis revealed that the reversed WMLs had 10\% higher $\lambda 1$ and $11 \%$ lower signal intensity on $b=0$ images compared with nonreversed WMLs. The ADC and $\lambda 2$ and $\lambda 3$ were not significantly different.

\section{Discussion}

Age-related WMLs are usually considered a progressive condition. ${ }^{4}$ A population-based study that analyzed 2 MR images separated by 5 years showed worsening in $28 \%$ of participants. ${ }^{3}$ The previously proposed mechanisms for progression included intermittent changes in cerebral perfusion pressure leading to incomplete infarction, ${ }^{5,6}$ leakage of toxic plasma components due to altered $\mathrm{BBB}$ permeability, ${ }^{7-9}$ and microembolic events. ${ }^{10}$ Each of these mechanisms would induce a progressive, but not reversible, condition. The present case with WML reversal must, therefore, have been induced by a different etiology.

There are a few clinical conditions in which transient brain edema is known to occur, which include hyperammonemia and posterior reversible encephalopathy syndrome. From the imaging perspective, the present case most closely resembles hyperammonemia. ${ }^{11}$ The previous report dealing with hyperammonemia illustrated reversal of punctate WMLs in a series 


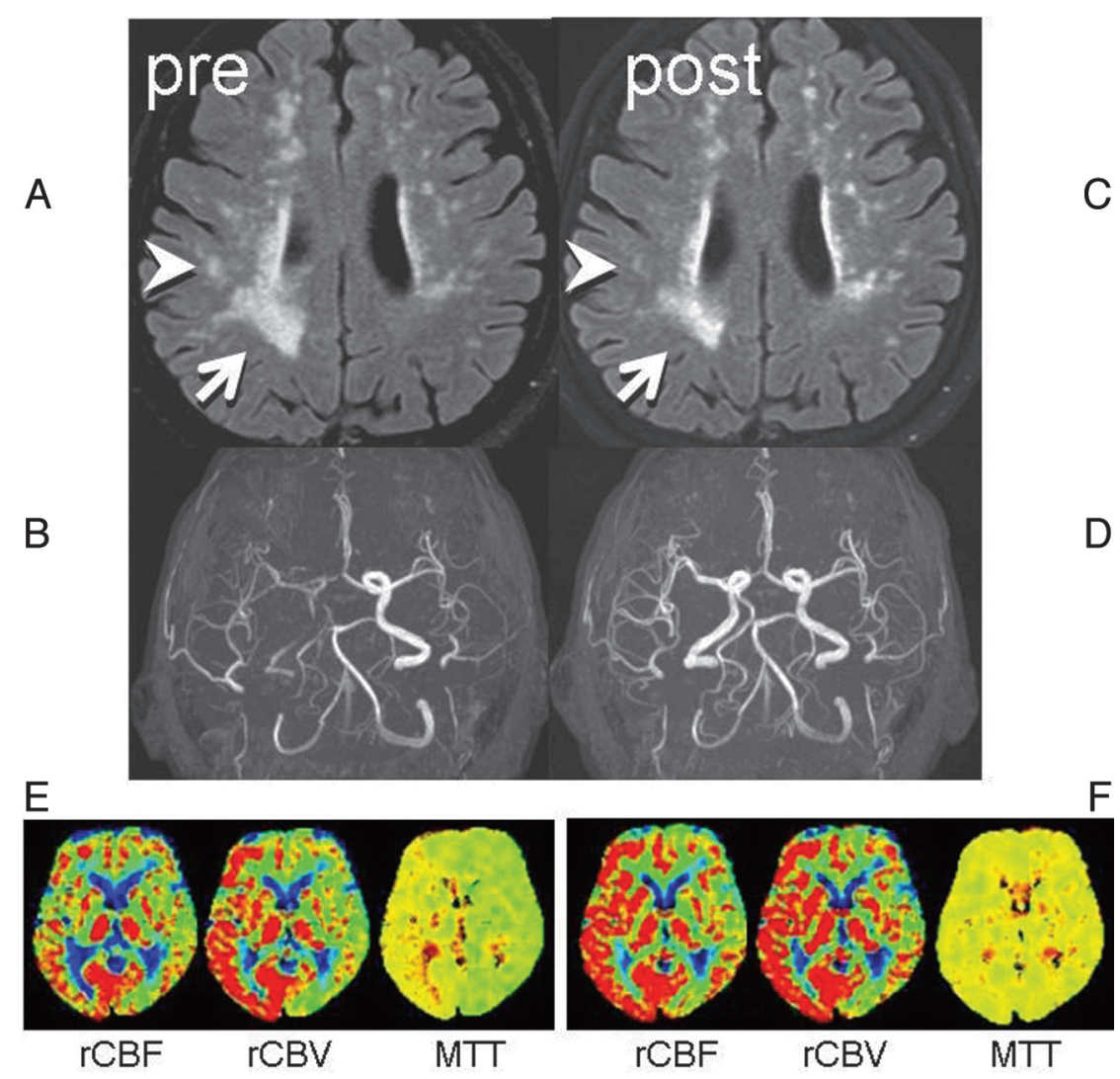

Fig 1. Two consecutive FLAIR images and MR angiography. A, Preprocedural FLAIR (delay time, $2200 \mathrm{~ms}$; TR/TE, 8000/100 ms) demonstrates WMLs. B, Preprocedural MR angiography (TR/TE, 30/2.3 ms) shows poor visualization of the right anterior circulation branches. C. Postprocedural FLAIR a week later shows partial WML resolution in the right hemisphere (arrow/arrowhead). D, MR angiogram shows restoration of flow in the right ICA. E, Preprocedural perfusion-weighted images show MTT elongation at the right anterior circulation territory with compensatory vasodilation, indicated by increased cerebral blood volume. F, After the procedure, the MTT elongation is normalized. Increased cerebral blood flow at the same territory indicates postprocedural hyperperfusion, but the patient remained asymptomatic.
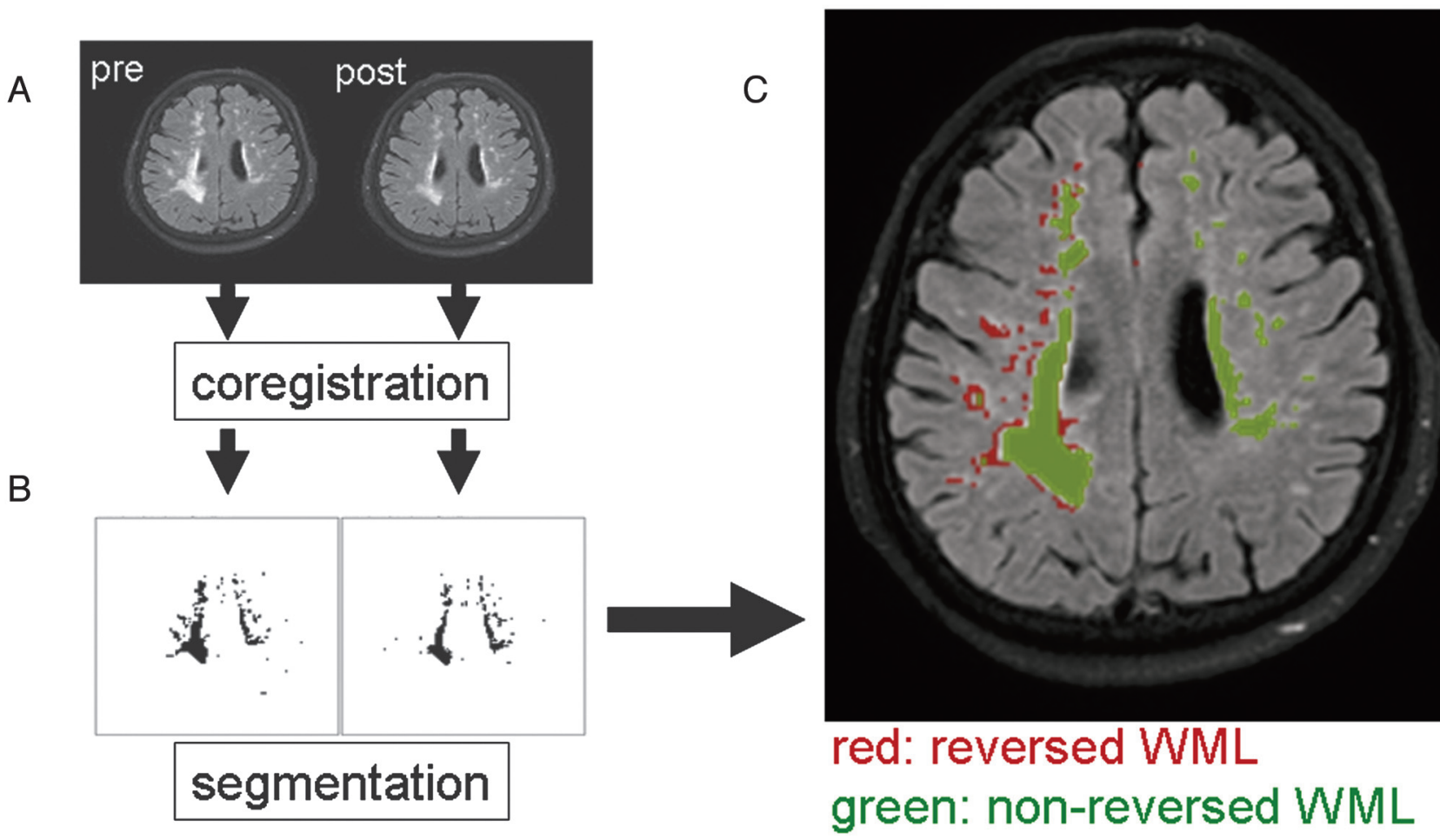

Fig 2. Image analysis on pre- and postprocedural images. $A$, Image coregistration was performed between the pre- and postprocedural FLAIR. $B$, Segmentation of the pixels with WMLs was then done. $C$, Subtraction images yielded pixels with/without reversal. Coregistration of these data onto the DWI/DTI (DWI: TR/TE, $\left.6000 / 88 \mathrm{~ms} ; b=1000 \mathrm{~s} / \mathrm{mm}^{2}\right)$ and $b=0 \mathrm{imaging}$ was performed to characterize the nature of the WMLs. 


\begin{tabular}{lcccc}
\hline \multicolumn{3}{l}{ Comparison between reversed and nonreversed WMLs } \\
\hline & \multicolumn{3}{c}{ WMLs } \\
\cline { 2 - 3 } & Reversed & \multicolumn{1}{c}{ Nonreversed } & $T$ Test \\
\hline $\mathrm{ADC} \times 10(-6)$ & 1.15 & & 1.28 & 0.127 \\
$\lambda 1 \times 10(-6)$ & 2.53 & $>$ & 2.29 & $0.001>$ \\
$\lambda 2$ or $\lambda 3 \times 10(-6)$ & 0.58 & & 0.58 & 0.336 \\
$b=0$ & 292 & $<$ & 328 & $0.001>$ \\
\hline
\end{tabular}

of patients with liver cirrhosis. ${ }^{11}$ These patients were treated with liver transplantation, after which the authors noted WML reversal. Hyperammonemia causes increased permeability through the BBB, which was considered to be the source of transient edema.

A case report described reversal of WMLs after brain infarction. ${ }^{12}$ This elderly patient had an acute brain infarction involving the occipital lobe. A follow-up MR imaging performed a year later revealed partial regression of WMLs. The authors of this report also speculated that this was due to transient disturbance of the $\mathrm{BBB}$, causing patchy white matter edema.

To investigate the underlying pathophysiology of WML reversal in the present case, we performed a retrospective image analysis. This revealed that the $\lambda 1$ and signal intensity on $b=$ 0 images were different, but by only $10 \%$. This finding may indicate that image-based differentiation between reversible and nonreversible WMLs may be challenging. A difference in $\lambda 1$ may indicate that nonreversed WMLs have axonal fragmentation or reduced axonal flow, but future pathologic correlation studies are needed to discover the true underlying mechanisms.

Aside from the pathophysiology, a few other clinical questions may arise. For instance, how often do we expect to see such reversal of WMLs after blood flow restoration and does this reversal indicate a successful treatment result? These issues would have sufficient impact on our clinical practice; thus, we have started a study to elucidate these issues.

\section{Acknowledgments}

We thank Drs Sachiko Yuen, Kentaro Akazawa, and Hiroyasu Sasajima for valuable comments on this study.

\section{References}

1. Hachinski VC, Potter P, Merskey H. Leukoaraiosis. Arch Neurol 1987;4:21-23

2. Wiszniewska M, Devuyst G, Bogousslavsky J, et al. What is the significance of leukoaraiosis in patients with acute ischemic stroke? Arch Neurol 2000;57: 967-73

3. Longstreth WT Jr, Arnold AM, Beauchamp NJ Jr, et al. Incidence, manifestations, and predictors of worsening white matter on serial cranial magnetic resonance imaging in the elderly. Stroke 2005;36:56-61

4. Streifler JY, Eliasziw M, Benavente OR, et al. Development and progression of leukoaraiosis in patients with brain ischemia and carotid artery disease. Stroke 2003;34:1913-16

5. Brun A, Englund EA. White matter disorder in dementia of the Alzheimer type: a pathoanatomical study. Ann Neurol 1986;19:253-62

6. Yamada K, Nagakane Y, Sasajima H, et al. Incidental acute infarcts identified on diffusion-weighted images: a university hospital-based study. AJNR Am J Neuroradiol 2008;29:937-40

7. Nakaji K, Ihara M, Takahashi C, et al. Matrix metalloproteinase-2 plays a critical role in the pathogenesis of white matter lesions after chronic cerebral hypoperfusion in rodents. Stroke 2006;37:2816-23

8. Caplan LR. Dilatative arteriopathy: what is known and not known. Ann Neurol 2005;57:469-71

9. Wardlaw JM, Sandercock PA, Dennis MS, et al. Is breakdown of the blood brain barrier responsible for lacunar stroke, leukoaraiosis, and dementia? Stroke 2003;34:806-12

10. Tejada J, Diez-Tejedor E, Hernandez-Echebarria L, et al. Does a relationship exist between carotid stenosis and lacunar infarction? Stroke 2003;34:1404-09

11. Rovira A, Mínguez B, Aymerich FX, et al. Decreased white matter lesion volume and improved cognitive function after liver transplantation. Hepatology 2007;465:1485-90

12. Moriya Y, Kozaki K, Nagai K, et al. Attenuation of brain white matter hyperintensities after cerebral infarction. [Letter] AJNR Am J Neuroradiol 2009;30: E43 